\title{
Emergency management of the COVID-19 pandemic in a Vascular Surgery Department of a large metropolitan hospital in Milan, Italy. Preparation, Escalation, De-escalation, Normal Activity
}

\author{
Roberto CHIESA ${ }^{1}$, Andrea Kahlberg ${ }^{2}$, Enrico Rinaldi ${ }^{2}$, and Daniele Mascia ${ }^{2}$ \\ ${ }^{1}$ Vita-Salute San Raffaele University, San Raffaele Scientific Institute \\ ${ }^{2}$ Vita-Salute University, IRCCS San Raffaele Scientific Institute
}

August 3, 2020

\begin{abstract}
During the first phase of COVID-19 pandemic in Italy, several strategies have been taken to deal with the pandemic outbreak. The Regional Authority of Lombardy remodeled the hospitalization system in order to allocate appropriate resources to treat COVID-19 patients and to identify "Hub/Spoke" hospitals for highly specialized medical activities. The Hubs hospitals were required to guarantee full time evaluation of all patients presenting with cardiovavascular diseases with an independent pathway for patients with suspect or confirmed COVID-19 infection. San Raffaele Hospital was identified as Hub for cardiovascular emergencies and the Vascular Surgery Department was remodeled to face this epidemic situation. Surgical treatment was reserved only to symptomatic, urgent or emergent cases. Large areas of the hospital were simultaneously reorganized to assist COVID-19 patients. During this period, 135 patients were referred to San Raffaele Vascular Surgery Department. COVID-19 was diagnosed in 24 patients and, among them, acute limb ischemia was the most common cause of admission. At this time, the COVID-19 trend is in decline in Italy and the local authorities reorganized the health care system in order to return to normal activities avoiding new escalations of COVID-19 cases. Several strategies have been taken to ensure the safety of the San Raffaele hospital, and maintaining potentially suspected patients with COVID-19 separated from other patients. The aim of this paper is to report the remodeling of the Vascular Surgery Department of San Raffaele Hospital as regards the strategies of preparation, escalation, de-escalation and return to normal activities during the COVID-19 pandemic.
\end{abstract}

Emergency management of the COVID-19 pandemic in a Vascular Surgery Department of a large metropolitan hospital in Milan, Italy.

Preparation, Escalation, De-escalation, Normal Activity

Authors:

Roberto Chiesa, MD, Andrea Kahlberg, MD, Enrico Rinaldi, MD, Daniele Mascia, MD

Affiliations:

Vascular Surgery, Vita-Salute University, IRCCS San Raffaele Scientific Institute,

Via Olgettina, 60, 20132, Milan, Italy.

Address for Correspondence and reprints:

Roberto Chiesa, MD

Vascular Surgery, Vita-Salute University, San Raffaele Scientific Institute,

Via Olgettina, 60, 20132, Milan, Italy. 
Fax: +39 02.2643.7148;

Phone: +39 02.2643.7130;

E-mail: chiesa.roberto@hsr.it

Word Count: 2528

Key-words: SARS-CoV-2, COVID-19, pandemic, vascular complications, emergencies.

\section{Introduction}

At the end of 2019, China reported a cluster of unexplained cases of viral pneumonia in Hubei province, where in approximately 30 days, spread from the city of Wuhan to the entire country, causing thousands of deaths. ${ }^{1,2}$ On February 2020, the World Health Organization (WHO) officially named this severe acute respiratory syndrome coronavirus 2 as coronavirus disease (COVID-19), caused by the 2019-nCoV virus, and on March 11, 2020, it has been officially declared a pandemic due to the constantly increasing number of cases all around the world. ${ }^{3}$ Globally, as of 28 June 2020, there have been 9.825 .539 confirmed cases of COVID-19, including 495.388 deaths in more than 180 countries, reported to WHO. ${ }^{4}$

In Italy, the first case tested positive for the new coronavirus was on February 21, 2020, at the Lombardy region, and since than, the number of reported COVID-19 patients rapidly spread all around the country. ${ }^{4}$ From the first confirmed case to 28 June 2020, there have been 239.961 positive patients with 34.708 deaths, being the region of Lombardy, a district in northern Italy with 10 million inhabitants, the most affected. ${ }^{4}$ In Italy, the peak of daily-confirmed cases was on March 21, 2020, with 6557 patients and the peak of deathconfirmed cases was on March 28, 2020, with 971 deaths related to COVID-19. ${ }^{4}$ Since then, the emergency response to the outbreak from the local health authorities started to flat and daily decrease the COVID-19 patients trend.

During the first phase of COVID-19 pandemic in Italy, the Regional Authority of Lombardy remodeled the hospitalization system in order to allocate appropriate resources to treat COVID-19 patients and to identify "Hub/Spoke" hospitals for highly specialized medical activities. ${ }^{2,5}$ San Raffaele Hospital - a large tertiary hospital and research center in Milan, Italy - was identified as Hub for cardiovascular emergencies and the vascular surgery department was remodeled to face this epidemic situation. ${ }^{2,5,6}$ Now that the COVID-19 trend is in decline, the local authorities reorganized the health care system in order to reopen and return to normal activities in order to avoid new escalations of COVID-19 cases. The aim of this paper is to report the remodeling of the Vascular Surgery Department of San Raffaele Hospital as regards the strategies of preparation, escalation, de-escalation and return to normal activities during the COVID-19 pandemic, as well as the vascular surgery patients data obtained during these phases.

\section{Before the storm}

Several strategies according to the anticipated needs for intensive care capacity for COVID-19 patients have been taken by the Government of Lombardy and local health authorities to deal with the pandemic outbreak. They decided to centralize surgical specialties activities, including major trauma, neurosurgery, interventional neuroradiology, interventional cardiology, cardiac surgery, and vascular surgery, creating a Hub/Spoke system in order to concentrate their resources for COVID-19 related cases. ${ }^{2,5,6}$ Vascular surgery Hubs in Lombardy were Monzino Hospital (Milan), Poliambulanza Hospital (Brescia), Legnano Hospital (Legnano) and San Raffaele Hospital (Milan).

The Hubs hospitals were required to guarantee full time evaluation of all patients presenting with known or suspected vascular surgery disease, even referred from the territorial emergency net or from Spoke hospitals; medical or surgical treatment of all cases presenting with symptomatic, urgent or emergent vascular disease, or deemed not able to wait for more than 30 days; at least 2 surgical teams full time available (composed by two surgeons, one anesthesiologist and two nurses), one of which leastwise on duty; and a dedicated independent pathway for patients with suspect or confirmed COVID-19 cases, separate to that one reserved for COVID-19 negative patients. 
San Raffaele Hospital initiated this new model on March 9, 2020, and surgical treatment was reserved only to symptomatic, urgent or emergent diseases. The elective surgical activity was rapidly reduced and large areas of the hospital were simultaneously reorganized to admit and assist COVID-19 patients. The general ward capacity was improved to a total of 279 beds dedicated to COVID-19 related cases and the number of intensive care unit (ICU) beds increased from 28 to 72 (18 to cardiology and cardiovascular surgery hub emergencies). ${ }^{6}$ A new COVID-19-free emergency room, dedicated only to cardiac and vascular cases, has been created and all patients admitted were tested for severe acute respiratory syndrome coronavirus 2 (SARS-CoV-2) by a nasopharyngeal swab and chest X-ray. Chest computed tomography (CT) scan was performed in selected cases. (Figure 1) All healthcare workers in these facilities were equipped with full personal protective equipment (PPE) and in case of suspected or confirmed COVID-19 in a patient requiring surgical treatment, PPE was provided for the entire surgical team. A dedicated COVID-19 operating room has been designated, and in case of general anesthesia, the surgical team waited outside the room while the patient was intubated. In those cases, anesthesiologists and surgeons wore double sterile gloves, surgical waterproof gowns, goggles for eye protection and N95 masks. In the postoperative period, COVID-19 patients were admitted to a COVID-19 dedicated ICU or to a dedicated ward.

As regarding Vascular Surgery reorganization, all outpatient activities (except urgencies) were blocked. Varicose veins surgeries were postponed indefinitely, and only considered emergent and urgent vascular surgeries were performed. Only aneurysms with high risk of rupture $(>6 \mathrm{~cm})$, symptomatic carotid lesions, critical limb-threatening ischemia (CLTI), and emergent cases of ruptured aneurysm and acute limb ischemia were performed.

As a drawback of this redefinitions of Italian care delivery, non-urgent procedures have been completely cancelled, at both inpatient and outpatient levels, and lots of diagnostic and surgical procedures were left behind. During the next phase, of escalation of COVID-19 cases, we observed more advanced grades of vascular diseases, such as gangrene or ruptured aneurysms, in which required more aggressive therapeutic methods. If treated in time properly, surely, some more aggressive procedures could be avoided. Data from patients with vascular disease treated afterwards are reported next, in the escalation phase.

\section{Fighting against COVID-19 does not mean abandoning our duties}

During the escalation period, 135 (91 males, $67 \%$ ) patients with a mean age $72 \pm 12$ years were referred to San Raffaele Vascular Surgery Department. COVID-19 was diagnosed in 24 patients (18\%), in 17 cases ad admission, whereas the remaining 7 patients were initially tested negative and became positive during subsequent hospitalization. Chest CT-scan was performed in 33 patients (24.0\%), presenting signs of interstitial pneumonia in 12 cases.

As regarding the demographic characteristics and preoperative risk factors, no significant differences were found between COVID-19 and non-COVID-19 patients, except for coronary artery disease that was more common in the non-COVID-19 group. The mean age of the COVID-19 patients was $69 \pm 12$ years, with 21 males (88\%) and in the non-COVID-19 group, $72 \pm 12$ years with 70 males $(63 \%)$.

Acute limb ischemia was the most common indication for admission, with thirty-two patients (24\%), which in 30 cases involved one inferior limb, in 4 cases one superior limb, in 2 cases both inferior limbs, and in one COVID-19 patient concomitant superior and inferior limb. Twenty-nine patients (21\%) presented with chronic inferior limb ischemia, and $4(3 \%)$ with already established irreversible inferior limb necrosis or gangrene. Futhermore, in COVID-19 patients, acute limb ischemia was the most common cause of admission, significantly more than in NON-COVID patients. Conversely, aortic and cerebrovascular disease were more commonly found in NON-COVID group

Aortic disease was the indication to admission in 26 patients (19\%), including 7 cases of abdominal aortic or iliac aneurysm (ruptured in 1 cases), 6 cases of endoleak in previous endovascular repair of abdominal aortic aneurysm (ruptured in 1 case), 5 cases of thoracoabdominal aortic aneurysm (ruptured in 1 case), 3 cases of acute type B aortic dissection, 2 cases of penetrating ulcer of the descending thoracic aorta, 1 case of descending thoracic aortic aneurysm, 1 case of abdominal aortic graft infection, and 1 case of aorto-duodenal 
fistula. Carotid stenosis was the indication to admission in 20 patients (15\%), symptomatic in 8 cases, and asymptomatic but preocclusive or due to an unstable plaque in 12 patients.

Interestingly, the time elapsed from the onset of symptoms to definitive treatment was significantly higher in COVID patients ( $43 \pm 71$ hours vs $17 \pm 24$ hours; $\mathrm{P}<0.0001$ ). Global in-hospital mortality was $2.2 \%$, and major adverse events were more present in COVID-19 patients. The first finding from this extensive experience is the raise of cases of acute limb ischemia in COVID patients. Previously reported experiences from all over the world emphasized the link between COVID-19 and microvascular inflammation, distal vasculitis and prothrombotic state. Linked to these findings, a "venous pattern" has also emerged: the increase of acute deep venous thrombosis cases in COVID-19 patients. ${ }^{5}$

\section{There is no calm after the storm}

From May 18, 2020, Government of Lombardy and local health authorities decided to start the process of reopening the region. At that time, the slowing trend in the number of deaths related to COVID-19 confirmed that the peak of the crisis has been left behind. At San Raffaele Hospital, the proportion of COVID-19 patients requiring ICU decreased over time from $17 \%$ to $7 \%$, with a significant drop in the mortality rate, from $24 \%$ to $2 \% .{ }^{7}$ Ciceri et al. highlight that a possible reduction of air pollution could be associated with decreased in factors associated with patient's morbidity, and the tracking of virus population diversity in time through SARS-CoV-2 mutations could potentially establish a correlation a viral fitness and virus attenuation. ${ }^{7}$

During this time, some strategies have been taken to ensure the safety of the San Raffaele hospital, and maintaining potentially suspected patients with COVID-19 separated from other patients. At the "reopening" phase, non-COVID care zones have been established, and all patients are screened for symptoms of COVID19, including temperature checks. These areas are separated from COVID-19 care zones, including in separate buildings and designated rooms with separate entrances. Besides that, all healthcare workers, before entering the hospital and initiate the shift are temperature checked. (Figure 2) Social distancing continued to be established inside the hospital, including minimizing the time in waiting areas with appropriately spacing chairs. The routine of cleaning and disinfecting continued with maximum level, as well as the use of PPEs. All individuals should wear a facemask.

A robust testing plan was developed, and all healthcare workers, including residents and all doctors, have been tested for SARS-CoV-2. Patients who were being scheduled for elective and high complex procedures are being tested before coming in for care. At San Raffaele, all surgical patients are required to be tested within 48 hours of their procedures and then self-isolated.

\section{Re-opening with new awareness}

Regulations and policies are continuing to adapt to a world in which COVID-19 exists. As temporary policies expire and new flexibilities emerge, healthcare facilities should include compliance as part of their reopening strategy. Now, the vascular surgery department is returning to normal activities, and resuming in-person care is essential to ensure the health of patients, regardless they have COVID-19, a chronic condition or other symptoms that require medical attention. Safety precautions and testing are essential to help care providers ensure safety and efficacy for those patients during this phase. A clear separation between COVID-19 and non-COVID-19 areas is required. Continuous testing of all admitted patients and temperature checks are mandatory.

Hospital facilities have been adapted for the resumption of activities in safety. The San Raffaele Hospital resumed ordinary activity according to the principle of graduality and safety after the sanitization of all spaces, both external and internal. Election hospitalization activities are gradually recovering, in addition to guaranteed continuity for urgent and emerging diseases. In compliance with the provisions issued by the General Welfare Directorate of the Lombardy Region on the containment of the epidemic, the San Raffaele Hospital followed some access rules. It is necessary to wear a surgical mask to access the environments for performing any type of procedure, and at the entrance, there is a body temperature control for everyone. Access to critical areas is currently not allowed, and a maximum of one companion is allowed and only if 
strictly necessary, or for the accompaniment of a minor, a disabled person or a person with frailty. The companion is also subject to all the above limitations and temperature control. Visits to hospital wards are contingent and limited to specific situations, subject to authorization by the head physician and the head nurse, and in any case in the maximum number of visitors per patient. Accompanying patients are prohibited from staying in the emergency waiting rooms unless specifically indicated, and in the waiting rooms it is mandatory to respect the spaces and seats made available to avoid close contacts.

Either patients or companions cannot access the San Raffaele Hospital if the person was subjected to the quarantine measure required by law unless specifically requested by the hospital to carry out the control swabs; if in the last days the person has manifested or still manifest symptoms of respiratory infection (cough, sore throat, headache) or have fever greater than $37.5^{\circ} \mathrm{C}$; if the person has tested positive for the SARS-CoV-2 swab or have had close contact with SARS-CoV-2 positive subjects in the past 14 days. If a patient has one of the above conditions, he can go to the hospital only with the authorization of a hospital reference doctor.

The hospital created an online and telephone system for booking visits. All visits and exams should be booked by these systems (by telephone or online through a dedicated website). Regarding the resumption of elective surgeries, all patients are tested for COVID-19 the day before using a drive-through system. The patient goes to a small lab facility and inside his car, is submitted to the SARS-CoV-2 swab test. Even if the test is negative, the patient must undergo a temperature check and a questionnaire about symptoms in the last 14 days. The entire outpatient path is separate from the inpatient path, with separate elevators, corridors, and nursing rooms. Only negative patients can be admitted to the wards.

Regarding the surgical procedure, all patients are submitted to a chest X-ray, and the COVID-19 swab-test is double-checked for the transport of the patient to the operating room. Throughout this process, the use of PPE is mandatory for all healthcare workers and patients. The ambulatories reduced the number of visits, and increased the time between them, to avoid contact between outpatients. An overwhelming number of visits postponed is being avoided, and therefore, outpatient activities are being held at extended hours and even on Saturdays.

Furthermore, besides clinical activities, continuously important research projects are being carried out in order to find new strategies and more effective therapies to better face this unprecedented health emergency in Italy. ${ }^{7}$

\section{Conclusion}

The COVID-19 pandemic will be remembered in Lombardy as a cataclysm that has affected the general population and especially patients of advanced age and worse general conditions. Adequate protection and wise allocation of human resources are very important during this period. The HUB / spoke system has proven to be a valid model in the management of ischemic emergencies. Government protocols should be continued, even when the number of cases decreases. A continuous international surveillance, co-operation, coordination and communication to effectively response to COVID-19 pandemic is crucial, because COVID19 reference centers could face similar issues and that could make them better prepared to deal with the pandemic COVID-19 and other epidemics.

\section{Author contribution}

Roberto Chiesa : Design, Drafting article, Critical revision of article, Approval of article

Andrea Kahlberg : Design, Data analysis, Drafting article, Critical revision of article, Approval of article

Enrico Rinaldi : Design, Data analysis, Drafting article, Critical revision of article, Approval of article

Daniele Mascia : Design, Data analysis, Drafting article, Critical revision of article, Approval of article

\section{References}


1. Wu Z, McGoogan JM. Characteristics of and Important Lessons From the Coronavirus Disease 2019 (COVID-19) Outbreak in China: Summary of a Report of 72314 Cases From the Chinese Center for Disease Control and Prevention. JAMA [Internet]. 2020;323:1239-1242. Available from: https://doi.org/10.1001/jama.2020.2648

2. Melissano G, Mascia D, Baccellieri D, Kahlberg A, Bertoglio L, Rinaldi E, Chiesa R. Pattern of vascular disease in Lombardy, Italy, during the first month of the COVID-19 outbreak. J. Vasc. Surg. 2020;

3. WHO Director-General's opening remarks at the media briefing on COVID-19: 11 March 2020. Published March 11, 2020. [Internet]. [cited 2020 Jun 17];Available from: https://www.who.int/dg/speeches/detail/who-director-general-s-opening-remarks-at-the-media-briefing-oncovid-19-11-march-2020

4. WHO Coronavirus Disease (COVID-19) Dashboard. Published June 28, 2020 [Internet]. [cited 2020 Jun 28]; Available from: https://covid19.who.int

5. Baccellieri D, Apruzzi L, Ardita V, Rinaldi E, Bertoglio L, Melissano G, Chiesa R. The "venous perspective" in Lombardia (Italy) during the first weeks of the COVID-19 epidemic. Phlebology. 2020;35:295-296.

6. Zangrillo A, Beretta L, Silvani P, Colombo S, Scandroglio AM, Dell'Acqua A, Fominskiy E, Landoni G, Monti G, Azzolini ML, Monaco F, Oriani A, Belletti A, Sartorelli M, Pallanch O, Saleh O, Sartini C, Nardelli P, Lombardi G, Morselli F, Scquizzato T, Frontera A, Ruggeri A, Scotti R, Assanelli A, Dagna L, RovereQuerini P, Castagna A, Scarpellini P, Di Napoli D, Ambrosio A, Ciceri F, Tresoldi M. Fast reshaping of intensive care unit facilities in a large metropolitan hospital in Milan, Italy: facing the COVID-19 pandemic emergency. Crit care Resusc J Australas Acad Crit Care Med . 2020;

7. Ciceri F, Ruggeri A, Lembo R, Puglisi R, Landoni G, Zangrillo A, COVID-BioB Study Group. Decreased in-hospital mortality in patients with COVID-19 pneumonia. Pathog Glob Health [Internet]. 2020;00:1-2. Available from: http://www.ncbi.nlm.nih.gov/pubmed/32584660

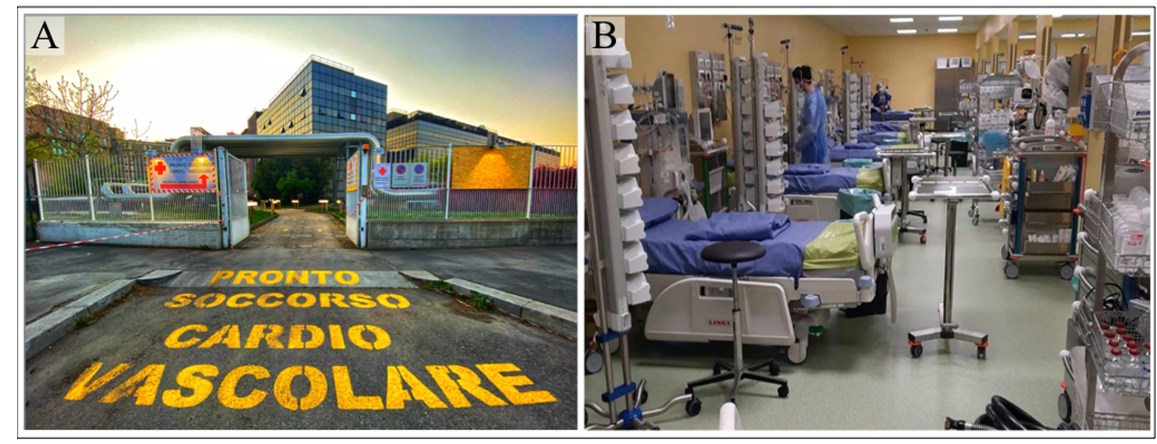




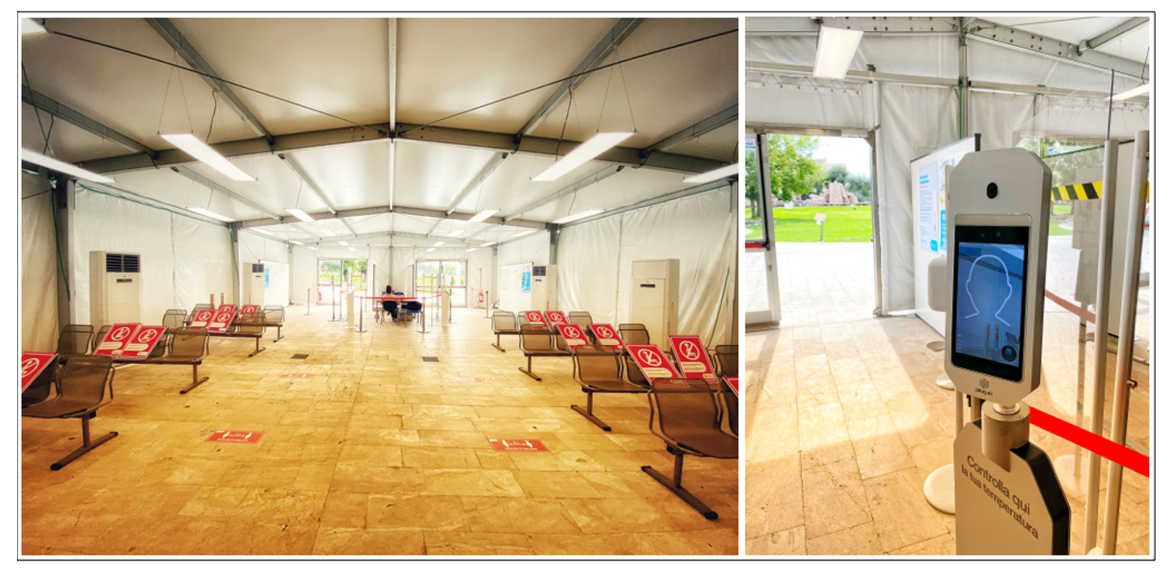

